\title{
Modelo de gestão do conhecimento para apoiar a prática clínica em uma unidade hospitalar
}

\author{
Knowledge management model to support clinical practice in a hospital \\ Modelo de gestión del conocimiento para apoyar la práctica clínica en un \\ hospital
}

\author{
Marcelo Ladislau da Silva | marceloladislaus@gmail.com \\ Universidade do Estado de Santa Catarina (UDESC). Florianópolis, Brasil. \\ Jordan Pauleski Juliani |jordanjuliani@gmail.com \\ Universidade do Estado de Santa Catarina (UDESC). Florianópolis, Brasil. \\ Júlio da Silva Dias |julio.dias@udesc.br \\ Universidade do Estado de Santa Catarina (UDESC). Florianópolis, Brasil.
}

\section{Resumo}

Estudos sobre gestão do conhecimento (GC) relatam como seu ciclo acontece na prática em empresas e indústrias, porém poucos a abordam como meio de aprimoramento das práticas clínicas em unidades hospitalares. O objetivo da pesquisa relatada neste artigo é contribuir para a redução dessa lacuna, por meio da proposta de um modelo de gestão do conhecimento para a melhoria da prática clínica. Após a realização de uma busca sistêmica da literatura, foram levantados os principais elementos de processos de GC para a construção de um modelo a ser utilizado na unidade hospitalar estudada. Tais processos serviram de base para realização do estudo de caso, no qual foram entrevistados os membros da equipe multiprofissional de atendimento ao paciente portador de fibrose cística (EMAPFC) de um hospital pediátrico de referência, do estado de Santa Catarina. Com base na análise dos resultados, foi elaborado um modelo de gestão do conhecimento que propicie melhorias no atendimento da unidade.

Palavras-chave: gestão do conhecimento; fibrose cística; prática clínica; unidade hospitalar; equipe multiprofissional.

\begin{abstract}
Studies on knowledge management report how, in practice, its cycle happens in businesses and industrial enterprises, but a few of them approach it as a means to improve the clinical practice in hospitals. The objective of the research related here is to contribute to bridge this gap, by means of a proposed model of knowledge management to improve the clinical practice. After conducting a systematic search of the literature, key elements of knowledge management processes were searched for the construction of a model to be used at the studied hospital. These processes were the base for the case study in which we interviewed members of the equipe multiprofissional de atendimento ao paciente portador de fibrose cística - EMAPFC (multi-professional team that treats the cystic fibrosis patient) at a pediatric hospital of reference, in the state of Santa Catarina. Based on the analysis of the results, we elaborated a model of knowledge management that provides improvements in the treatment for that disease at that hospital.
\end{abstract}

Keywords: knowledge management; cystic fibrosis; clinical practice; hospital; multi-professional team. 


\section{Resumen}

Estudios sobre la gestión del conocimiento (GC) describen como se produce su ciclo en la práctica en negocios y industrias, pero pocos la abordan como un medio para la mejora de las prácticas clínicas en hospitales. El objetivo de la investigación descripta en este artículo es contribuir para reducir esa espacio vacío, mediante la propuesta de un modelo de gestión del conocimiento para mejorar la práctica clínica. Después de realizar una búsqueda sistemática de la literatura, buscamos descubrir los elementos clave de procesos de GC para la construcción de un modelo de gestión del conocimiento para lo utilizar en el hospital estudiado. Esos procesos fueron la base para la realización del estudio de caso en el que entrevistamos los miembros de la equipe multiprofissional de atendimento ao paciente portador de fibrose cística - EMAPFC (equipo multiprofesional que atiende los pacientes con fibrosis quística) de un hospital pediátrico de referencia, en el estado de Santa Catarina. Basando en el análisis de los resultados, elaboramos un modelo de gestión del conocimiento que proporcione mejoras en el tratamiento para esa enfermedad en aquel hospital.

Palabras clave: gestión del conocimiento; fibrosis quística; práctica clínica; hospital; equipo multiprofesional.

Contribuição dos autores: Os pesquisadores citados fizeram contribuições intelectuais substanciais para a elaboração do presente artigos submetido à apreciação para publicação, portanto são responsável pelo documento analisado. Os três autores fizeram contribuições substanciais para a concepção ou desenho da obra; sendo que a aquisição, análise ou interpretação dos dados para o trabalho ficou sob-responsabilidade do pesquisador Marcelo Ladislau da silva, e a elaboração do trabalho e revisão crítica do conteúdo intelectual foi compartilhada pelos três autores. Também foi discutido entre os autores o texto construído, após a revisão solicitada pelos avaliadores deste periódico para aprovação final da versão a ser publicada onde todos participaram desta nova escrita. Os autores citados são responsáveis por todos os aspectos do trabalho no sentido de garantir que as questões relacionadas à precisão ou integridade de qualquer parte do trabalho sejam devidamente investigadas e resolvidas.

Declaração de conflito de interesses: não houve.

Fontes de financiamento: Todos os custos referentes a todas as etapas de concepção e execução do projeto foram custados pelos autores, não implicando em custos para os participantes bem como para as instituições envolvidas (Universidade e Unidade Hospitalar).

Considerações éticas: $O$ projeto de pesquisa que deu origem ao presente artigo foi submetido a apreciação do Comitê de Ética do Hospital Infantil Joana de Gusmão sobre o registro CAAE: 45238115.5.0000.5361, recebendo aprovação no parecer número 1.081 .725 .

Agradecimento/Contribuições adicionais: Agradecemos a todos os participantes e instituições por contribuir com o desenvolvimento do conhecimento científico.

Histórico do artigo: Submetido: 26.mar.2016 | Aceito: 09.set.2016 | Publicado: 23.dez.2016.

Apresentação anterior: Artigo com texto inédito, não tendo sido apresentado anteriormente.

Licença CC BY-NC atribuição não comercial. Com essa licença é permitido acessar, baixar (download), copiar, imprimir, compartilhar, reutilizar e distribuir os artigos, desde que para uso não comercial e com a citação da fonte, conferindo os devidos créditos de autoria e menção à Reciis. Nesses casos, nenhuma permissão é necessária por parte dos autores ou dos editores. 


\section{Introdução}

No contexto atual da sociedade, o conhecimento destaca-se como fator produtivo de competitividade. Nesse sentido, práticas de gestão do conhecimento (GC) vêm sendo implementadas em organizações públicas e privadas da área da saúde, com o intuito de propiciar melhorias na qualidade dos produtos e serviços prestados ao público atendido, bem como de tornar mais eficientes e eficazes os processos e serviços dependentes do conhecimento existente nesses locais. De acordo com Davila, Fraga, Diana e Spanhol ${ }^{1}$ para que a GC seja incorporada, ela deve primeiramente ser reconhecida como estratégia de negócio, de modo a envolver todos os indivíduos da organização. Considera-se que processos e iniciativas de GC têm gerado ações e debates crescentes em vários países, contribuindo com melhorias em diferentes dimensões, como: cultura, estratégia, estrutura e tecnologia.

Esse momento introduziu novas expressões no cotidiano organizacional. É possível observar o uso de locuções adjetivas tais como 'era da informação', 'era do conhecimento' ou 'era da comunicação'.

O objetivo maior desse período, seja qual for a denominação considerada, é potencializar a construção de novos conhecimentos. Assim, o conhecimento assume papel de destaque na sociedade atual, impactando diretamente o desenvolvimento individual e organizacional. Na sociedade do conhecimento, para as organizações sobreviverem e competirem, faz-se necessário um sistema de $\mathrm{GC}^{2}$.

$\mathrm{Na}$ área da saúde, não é diferente. A crescente quantidade de conhecimento gerado nas relações de troca entre a equipe multidisciplinar, formada por médicos, enfermeiros e outros profissionais que atuam na assistência ao paciente, traz às organizações de saúde recursos que podem ser armazenados e recuperados para sustentar e manter a eficácia da instituição³.

De acordo com Lui, Cheng, Chao e Tseng 4 , cada vez mais as organizações de saúde buscam promover a GC de modo a utilizar de forma eficaz o conhecimento necessário para melhor atender ao paciente. Conforme Nonaka e Takeuchi ${ }^{5}$, a GC na área da saúde possibilita o desenvolvimento dos conceitos relacionados à evolução da tecnologia na saúde, da medicina baseada em evidências científicas, da gestão clínica, da economia da saúde e da investigação dos serviços sanitários. Assim, esses autores ponderam que práticas de GC contribuem para a prestação de serviços de saúde com mais qualidade e na quantidade necessária à demanda da sociedade.

Organizações de saúde, especialmente as públicas, em escala global carecem de soluções via o

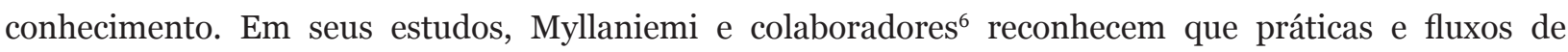
conhecimento existem em vasta quantidade nas organizações da saúde, mas estas carecem de meios para analisar, processar e gerir o conhecimento. Estudos sobre a GC em unidades hospitalares (UHs) podem ser considerados incipientes e, quando se faz uma leitura do cenário brasileiro com base na literatura existente, percebe-se que ainda são escassos como destacam Rowley ${ }^{7}$, Borga e Kliemann Neto ${ }^{8}$. Para os autores, são poucas as pesquisas sobre $\mathrm{GC}$ realizadas com foco na melhoria da qualidade dos serviços prestados na área da saúde; geralmente, as pesquisas feitas estão relacionadas à redução de custos. Portanto, é possível inferir a necessidade de se buscar respostas nas diferentes áreas de atuação, principalmente nas relacionadas às necessidades básicas, como saúde e educação. Para isso, também foi levado em consideração o fato de que, segundo Batista ${ }^{9}$, a administração pública deve criar possibilidades de aumento da efetividade da prestação de seus serviços para a sociedade a que serve.

Considerando que as organizações hospitalares são complexas e necessitam acompanhar constantemente as tecnologias, os processos e a qualidade dos serviços prestados pelo seu corpo clínico e administrativo, realizou-se uma pesquisa, com o objetivo de contribuir para a melhoria de serviços e processos em uma unidade hospitalar (UH) com a resposta à problemática da pesquisa: como implementar um modelo de gestão do conhecimento para apoiar a prática clínica em uma UH pediátrica especializada em fibrose cística? 


\section{Conhecimento}

Diferentemente da informação, o conhecimento diz respeito a crenças e valores. Enquanto a informação é meramente uma matéria-prima, estruturada em dados e números, de aplicabilidade não provada, o conhecimento representa o propósito de uso da informação por meio de relações referentes a situações específicas ${ }^{4,10}$

Para Macedo e colaboradores ${ }^{11}$, o conhecimento é "[...] o conjunto completo de informações, dados, relações que levam as pessoas a tomar decisões, a desempenhar atividades e a criar novas informações ou conhecimentos".

Os autores Ponchirolli e Fialho ${ }^{2}$ e Mattera ${ }^{12}$ complementam apontando o conhecimento como resultante de um processo individual, cognitivo e subjetivo da pessoa com base em informações e experiências utilizadas para resolver determinados problemas. Fazem parte desse conjunto: teorias e práticas, regras e instruções de como agir.

Assim, o conhecimento é constituído por uma mescla entre informações adquiridas, crenças individuais existentes e experiências vivenciadas, formando algo único (pessoal) que é passível de ser transmitido a outros por meio de artefatos digitais ou analógicos ou que pode ainda ser constatado pela observação. A informação se torna conhecimento quando é aplicada a um contexto. O conhecimento se constitui do significado e valor que agrega para uma pessoa, podendo ser subjetivo, ilimitado e estar constantemente em construção.

Davenport e Prusak ${ }^{13}$ conceituam conhecimento como uma mistura fluida de experiências, valores, informação contextual e insight, proporcionando uma estrutura para avaliação e incorporação de novas experiências e informações. Para os autores, o conhecimento tem origem na mente do indivíduo como ser cognoscente, alguém que pensa e constrói conhecimento. Quando se trata de conhecimento organizacional, geralmente este é registrado em documentos e repositórios, como também em rotinas, processos e normas organizacionais. Para Gnecco Junior ${ }^{14}$, “o conhecimento é o único recurso que aumenta com a utilização” e que contribui para a obtenção de vantagens competitivas e individuais dos que fazem uso dele, promovendo o crescimento da empresa ${ }^{5}$.

O conhecimento é atualmente um grande diferencial competitivo nos países e se consolida como fator-chave para que as organizações sobrevivam e se mantenham competitivas, portanto precisa ser identificado e gerenciado ${ }^{11,14,15}$. Segundo Nonaka e Takeuchi ${ }^{16}$, ele ocorre em três níveis: individual, grupal e organizacional. Segundo a teoria de criação do conhecimento proposta por esses autores, ele pode ainda ser classificado de duas formas: conhecimento tácito e conhecimento explícito.

O primeiro é difícil de ser externalizado ou disseminado, pois se refere ao conhecimento pessoal do indivíduo; muitas vezes é difícil sua formalização. Normalmente está registrado na mente da pessoa, com base em suas ações e suas experiências. Já o explícito, é expresso em palavras, números ou sons e compartilhado por meio de dados, fórmulas, recursos visuais, produtos e manuais ${ }^{5}$.

Corroborando essa noção, Von Krog, Ichijo e Nonaka ${ }^{17}$ relatam que o conhecimento explícito é o que se articula em linguagem formal, é sistematizado e comunicado, através de livro-texto, por exemplo, ou expresso formalmente através de um sistema de símbolos que possibilita sua fácil comunicação ou difusão em formato digital ou analógico de modo que se torne disponível aos interessados ${ }^{16,18,19}$.

O conhecimento tácito, por sua vez, tem a característica de ser de difícil articulação através da linguagem formal. É pessoal; está incorporado à experiência do indivíduo, articulado por suas crenças, seus valores, suas perspectivas, competências, habilidades e emoções. Assim, Nonaka e Takeuchi ${ }^{5}$ afirmam que esse tipo de conhecimento não é de fácil explicação e visibilidade; pelo contrário, é pessoal, difícil de formalizar e transferir. Palpites e intuições compõem o conhecimento tácito, e ele está profundamente enraizado 
e envolvido nas ações e experiências corporais do indivíduo, bem como nos seus sentidos e valores incorporados. Com base nessa discussão, elaborou-se o Quadro 1, para uma melhor compreensão.

Quadro 1 - Conhecimento tácito e explícito: características

\begin{tabular}{|l|l|}
\hline Conhecimento tácito & Conhecimento explícito \\
\hline Difícil codificação & Codificável \\
\hline Acesso restrito & Acesso facilitado \\
\hline Difícil de ser compartilhado & Fácil de ser compartilhado \\
\hline $\begin{array}{l}\text { Relacionado ao contexto prático do } \\
\text { indivíduo }\end{array}$ & Baseado na racionalidade \\
\hline Aprendizado baseado na ação & $\begin{array}{l}\text { Aprendizado adquirido por meio de uma } \\
\text { estruturação formal e sistemática - estudo formal }\end{array}$ \\
\hline
\end{tabular}

Fonte: Elaborado pelos autores (2016).

Nas organizações, os ativos de conhecimento podem ainda ser classificados como conhecimento individual (quando intimamente ligado ao indivíduo que o detém) ou conhecimento coletivo (não redutível ao indivíduo $)^{20}$.

Corroborando essa ideia, Nonaka e Peltokorpi ${ }^{21}$ apontam que a criação de conhecimento se inicia tanto nos indivíduos, capazes de adquirir e processar o conhecimento tácito, quanto na interação com o ambiente e a organização, e geram o conhecimento organizacional.

Alvarenga Neto $^{22}$ diz que o conhecimento pode ser compartilhado ou transferido em um ambiente organizacional que estimule esse comportamento, por meio da interação e valorização do conhecimento individual dos integrantes da empresa.

É relatado na obra de Nonak e Takeuchi ${ }^{16}$ que o conhecimento organizacional é a capacidade de criação e disseminação de conhecimento no âmbito organizacional, para incorporá-lo a produtos, serviços e sistemas de modo a se obter vantagem competitiva sustentável.

Contudo, Dalkir ${ }^{23}$ alerta para o fato de que, independentemente do tipo de conhecimento, este só terá valor se aplicado à tomada de decisões. Esse pensamento é corroborado por Cavalcante ${ }^{24}$ ao descrever que o conhecimento está associado à criação, construção, manutenção e mudança que as pessoas fazem no ambiente em que atuam. Portanto, para identificar, utilizar e armazenar os ativos de conhecimento existentes em uma organização, é preciso fazer a sua gestão por meio de técnicas e processos que contemplem o ciclo de vida do conhecimento. Segundo Ponchirolli e Fialho² ${ }^{2}$, na sociedade do conhecimento para as organizações sobreviverem e competirem faz-se necessário um sistema de GC. Neste sentido, no item a seguir, discute-se a gestão do conhecimento, seus processos, facilitadores e limitadores, além de se apresentar o tema à luz das práticas da área da saúde.

\section{Gestão do conhecimento}

O conceito de gestão do conhecimento não é novo. É relatado por Pacheco ${ }^{25}$ que a GC ainda se encontra em desenvolvimento nos dias atuais, embora tenha surgido no início da década de 1990, quando passou a fazer parte da estratégia empresarial e a ser entendida como fator gerador de valor para a organização. Para Bem, Prado e Delfino ${ }^{26}$, vivemos na economia do conhecimento e por isso este assume papel estratégico central para o desenvolvimento da competitividade nas organizações, sejam públicas ou privadas. Na administração pública, a GC deve estar atrelada a princípios básicos do serviço público, como eficiência, 
qualidade, efetividade social, e também aos princípios constitucionais da legalidade, impessoalidade, moralidade, publicidade e eficiência9.

Diferentes definições de GC são propostas na literatura, e diversas discussões têm por base as sucessivas passagens do conhecimento tácito para o conhecimento explícito e vice-versa, na chamada espiral do conhecimento, proposta por Nonaka e Peltokorpi ${ }^{21}$. Para os autores, a GC é a atividade de divulgar e explicitar os saberes implícitos nas práticas individuais e coletivas das organizações, em um movimento espiralado que evolui a partir dos níveis inferiores e promove a construção de novos conhecimentos.

Para que esse ciclo aconteça, são necessárias a verbalização e a modelagem do conhecimento sob a forma de documentos, manuais, histórias, metáforas ${ }^{21}$. Para Nonaka e Takeuchi ${ }^{16}$, a GC está relacionada à conversão do conhecimento explícito em tácito, e esse processo de conversão se dá em quatro momentos, conforme o quadro 1: socialização, externalização, combinação e internalização.

De acordo com Valentim ${ }^{27}$ a GC pode ser interpretada como o conjunto de atividades estruturadas para desenvolver a cultura organizacional e a comunicação entre os atores presentes, visando propiciar a criação, a aquisição, o compartilhamento e a utilização do conhecimento, bem como mapear os fluxos informais para a formalização, com o intuito de converter o conhecimento individual (tácito) em explícito, contribuindo para a resolução de problemas e a geração de novas ideias.

A gestão do conhecimento, segundo Mattera ${ }^{12}$ oferece à organização um conjunto de práticas, metodologias e ferramentas para o aperfeiçoamento de sua gestão visando à excelência por meio da aplicação efetiva do conhecimento organizacional, o que contribui para a melhoria contínua e a inovação de diferentes processos, como: aquisição, criação, armazenamento, compartilhamento, utilização e reutilização do conhecimento em uma organização.

Para Ponchirolli e Fialho², GC implica: “[...] maximizar e alavancar o potencial das pessoas. É a estratégia que converte os bens intelectuais das organizações - tanto informações quanto talentos dos membros - em maior produtividade, novo valor e aumento da competitividade".

Complementando, Probst, Raub e Romhardt ${ }^{20}$ afirmam que "a gestão do conhecimento consiste em um conjunto integrado de intervenções que aproveitam as oportunidades para dar forma à base de conhecimento". Para os autores, a GC pode ser aplicada no nível individual, grupal ou organizacional. Ao trabalhar GC, Rowley ${ }^{7}$ apresenta uma cadeia de valor do conhecimento que consiste em quatro elementos essenciais:

- Dados: base para a informação. Aquilo que pode ser captado pelos órgãos dos sentidos humanos. Não tem valor agregado e de forma isolada é pouco significativo.

- Informação: base para o conhecimento. Agrega valor aos dados através da contextualização.

- Conhecimento: processo cognitivo que mistura experiência, valor, verdade e discernimento.

- Competência: capacidade de inovar, conhecimento internalizado.

No setor público, a GC tem ainda outras especificidades, como destaca Batista9. Para o autor, a GC consiste em:

[...] um método integrado de criar, compartilhar e aplicar o conhecimento para aumentar a eficiência; melhorar a qualidade e a efetividade social; e contribuir para a legalidade, impessoalidade, moralidade e publicidade na administração pública e para o desenvolvimento brasileiro.

A Asian Productivity Organization (APO) destaca que o ponto de partida da GC é entender a visão, missão, direção e os objetivos da organização com base nos elementos: pessoas (liderança), processos e tecnologia. Essa compreensão permite uma implementação de GC bem-sucedida ${ }^{28}$. 
Considerando que o objetivo deste artigo é apresentar um modelo de GC para uma UH, torna-se relevante destacar que o processo de GC é constituído por práticas de utilização do conhecimento na gestão, na implementação e na melhoria dos processos de trabalho ${ }^{28}$. Assim, a GC envolve um conjunto de processos do conhecimento, por meio de pessoas e tecnologias. Segundo Sumet, Suwannapong, Howteerakul, Thammarat ${ }^{29}$, a GC na área médica apresenta três fases, a saber: preparação, execução e avaliação. Na primeira fase, ou preparação, é feita uma introdução ao tema e explicada a importância da GC. Na etapa seguinte, a da execução, realizam-se atividades para compartilhar o conhecimento e a experiência no grupo. E, por fim, na fase de avaliação, que no estudo aconteceu após um mês da implementação da GC, o grupo envolvido é reunido para avaliar a contribuição e a prática da proposta. Depois de implementar um modelo em uma UH, os autores perceberam o aumento da satisfação pessoal no trabalho, bem como da satisfação do paciente com os serviços prestados.

Para Elwyn, Taubert e Kowalczuk ${ }^{30}$ assim como para Laihonen ${ }^{31}$, a prática de GC se dá a partir do processo de transferência do conhecimento. Para os autores, desempenhar essa ação ajuda a superação de barreiras. Ao compartilhar o conhecimento, é possível encontrar algumas das respostas que estão sendo procuradas, inclusive com evidências empíricas e já testadas. Para que a transferência do conhecimento seja viável, duas ações podem ser consideradas essenciais. São elas: decisão de transferir; e uso do conhecimento transferido para alcançar o desempenho satisfatório.

Com base nesses estudos, observa-se que as práticas de GC, independentemente da área de atuação, recaem sobre o uso do conhecimento existente como meio de criação de valor que contribui para a organização e para todos os envolvidos. Com base no estudo realizado, considerou-se, para fins desta pesquisa, conforme identificado de modo recorrente na literatura, que os processos de GC envolvem seis etapas, conforme mostra o Quadro 2.

Quadro 2 - Processos de gestão do conhecimento

\begin{tabular}{|l|l|c|}
\hline \multicolumn{1}{|c|}{ Processos } & Relações por analogia das palavras & Ocorrência \\
\hline Compartilhamento & Socialização e externalização & 7 vezes \\
\hline Utilização & Uso, aplicação e combinação & 7 vezes \\
\hline Desenvolvimento & Criação & 6 vezes \\
\hline Retenção & Armazenamento e internalização & 5 vezes \\
\hline Aquisição & Captura & 4 vezes \\
\hline Identificação & --- & 3 vezes \\
\hline
\end{tabular}

Fonte: Elaborado pelos autores (2016).

Contudo, algumas limitações podem ser encontradas na implementação e nas práticas de GC, o que exige o envolvimento e análise do contexto em que se atua.

\section{Procedimento metodológico}

Esta pesquisa, realizada visando em parte a uma dissertação de mestrado, caracteriza-se como aplicada, pois tem como objetivo final apresentar soluções para as práticas organizacionais no contexto hospitalar. Classifica-se ainda como exploratório-descritiva. A abordagem do estudo baseia-se na natureza do problema pesquisado, tendo sido realizada uma análise qualitativa para explorar e entender o significado que os indivíduos atribuem à situação em estudo ${ }^{22}$. 
Os métodos de pesquisa utilizados para coleta de dados foram: levantamento bibliográfico; levantamento documental e estudo de caso. Inicialmente, para verificação e consolidação do problema a ser pesquisado, centrou-se em um levantamento bibliográfico, que, segundo Creswell ${ }^{32}$, tem o intuito de localizar, nas diferentes bases de análises, estudos científicos, estudos conceituais ou ponderações que permitam reflexões científicas sobre um determinado tema. Para fazer esse levantamento, buscou-se a literatura clássica de GC, e para identificar a relação deste tema com a área médica foi realizada uma pesquisa no banco de dados on line Scopus, selecionado por ser considerado o maior da literatura científica revisada por pares nas áreas de ciência, tecnologia, medicina, ciências sociais e artes e humanidades.

A busca foi realizada utilizando as expressões: 'gestão do conhecimento', 'modelo', 'saúde', 'médico' e 'hospitalar'. As expressões foram utilizadas em inglês, por ser uma busca em base internacional, com os seguintes termos: 'knowledge management' AND 'model' AND 'health' OR 'medical' OR 'hospita*'. Para o termo 'hospital', utilizou-se o truncador asterisco (*) no final, de modo a buscar a palavra com suas variações. Dessa busca, resultaram 882 documentos, dos quais ao separar os artigos relacionados a diferentes áreas da saúde, ciências sociais e multidisciplinares, foram obtidos 158 trabalhos. Após filtrá-los de acordo com o critério últimos dez anos, restaram 141 trabalhos. Em seguida, foram selecionados os artigos nos idiomas inglês, português e espanhol, o que resultou em 137 trabalhos. Na sequência, fez-se uma seleção por título, chegando a 36 artigos alinhados com os objetivos da pesquisa. Após a leitura de seus resumos, considerou-se que apenas 15 poderiam dar suporte ao tema da pesquisa e, a partir de então, realizou-se a leitura integral dos textos. O resultado foi uma amostra final de apenas seis artigos selecionados para contribuir diretamente com a fundamentação teórica deste estudo.

Considera-se que a busca no banco de dados on line selecionado contribuiu para essa pesquisa; contudo foi necessário ampliar a busca por meio da localização e seleção de obras de outros autores na área em estudo. Ainda no que tange ao método de pesquisa, em um segundo momento, trabalhou-se também com dados documentais com base no estudo de caso realizado num hospital público pediátrico.

Utilizou-se o estudo de caso como a estratégia de investigação com o objetivo de construir uma explicação sistemática dos fatos existentes no contexto analisado, no qual as múltiplas variáveis encontradas são contextualizadas com o apoio da estatística - os dados passam a ser representados sob a forma de tabelas, quadros, gráficos estatísticos - e por meio de uma análise descritiva que os caracteriza. Neste artigo, a análise está relacionada a uma especialidade denominada fibrose cística, que é uma subespecialidade da pneumologia, de uma unidade pública hospitalar do estado de Santa Catarina, o Hospital Infantil Joana de Gusmão (HIJG). Essa escolha deu-se pela proximidade do pesquisador com a equipe bem como pela organização da equipe que tem a preocupação com o tema de estudo, ou seja, com o conhecimento como ativo estratégico para o atendimento do paciente.

Com base no ambiente de análise e nas variáveis a serem observadas, definiu-se como instrumento de coleta de dados a entrevista semiestruturada para identificar quais processos de GC são comumente utilizados na prática clínica da UH em estudo. Optou-se por esse tipo de entrevista porque ela permite certa flexibilidade na abordagem do entrevistado. As entrevistas foram individuais, gravadas em meio digital, transcritas logo após terem sido realizadas. $\mathrm{O}$ resultado foi organizado em uma matriz dialógica problematizadora organizada conforme a preocupação temática. O local da entrevista foi escolhido pelos participantes segundo a sua disponibilidade de dia e horário.

Para analisar, utilizou-se como referência a análise do discurso do sujeito coletivo (DSC), de Lefevre e Lefevre ${ }^{33}$. Esse procedimento reúne em um discurso-síntese a ideia-chave (ICH) e a ideia central (IC). "ICH são pedaços, ou trechos, ou segmentos, contínuos ou descontínuos do discurso, que devem ser selecionados pelo pesquisador e que revelam a essência do conteúdo do depoimento ou discurso [...]"33. "IC é um nome ou expressão linguística que revela e descreve da maneira mais sintética e precisa possível o sentido ou os sentidos das ICH [...] As IC são o que o entrevistado quis dizer (ou o quê, sobre o quê) e as ICH como isso foi dito"33. 
O levantamento e a análise dos dados não procuraram restringir nem esgotar os atributos que poderiam ser encontrados em diferentes perspectivas observadas nos processos de GC na área pública hospitalar. No entanto, por se tratar de uma realidade específica, a impossibilidade de generalização dos resultados limita o estudo. Contudo, mesmo diante das possíveis limitações, o método adotado foi considerado o mais apropriado para alcançar o objetivo da pesquisa.

\section{Resultados da pesquisa}

A proposição de um modelo de GC para a UH em estudo tem o intuito de contribuir com a prática clínica, uma vez que o conhecimento existente, adquirido e desenvolvido pode ser potencializado se compartilhado, promovendo um atendimento clínico de maior qualidade.

Entende-se, neste estudo, modelo como “[...] uma abstração formal e, como tal, passível de ser manipulada, transformada e recomposta em combinações infinitas $[. . .]^{34}$. Com base nessa definição e considerando a última questão da entrevista realizada com os membros da equipe multiprofissional de atendimento ao paciente portador de fibrose cística (EMAPFC), concluiu-se que a GC na UH em estudo, a partir da realidade observada, deve contemplar os seis principais processos, já apontados no Quadro 2: identificação do conhecimento, indicado como primeiro processo; aquisição do conhecimento, como segundo; desenvolvimento do conhecimento, como terceiro; utilização do conhecimento, como quarto; compartilhamento e retenção do conhecimento, citados com a mesma frequência nas entrevistas, respectivamente definidos como quinto e sexto processos.

Dessa forma, a partir da análise dos resultados, identificou-se o ciclo dos processos de GC relevante para a UH em análise, a partir da literatura. Assim, com base nos resultados, elaborou-se a Figura 1 referente ao ciclo dos processos de GC identificados na UH.

Esse modelo de GC para UH contempla os seis principais processos identificados a partir da literatura evalidados por meio da pesquisa realizada com entrevista junto aos integrantes da EMAPFC. O objetivo é contribuir para a melhoria da prática clínica, uma vez que o conhecimento é o ponto central para um atendimento de qualidade.

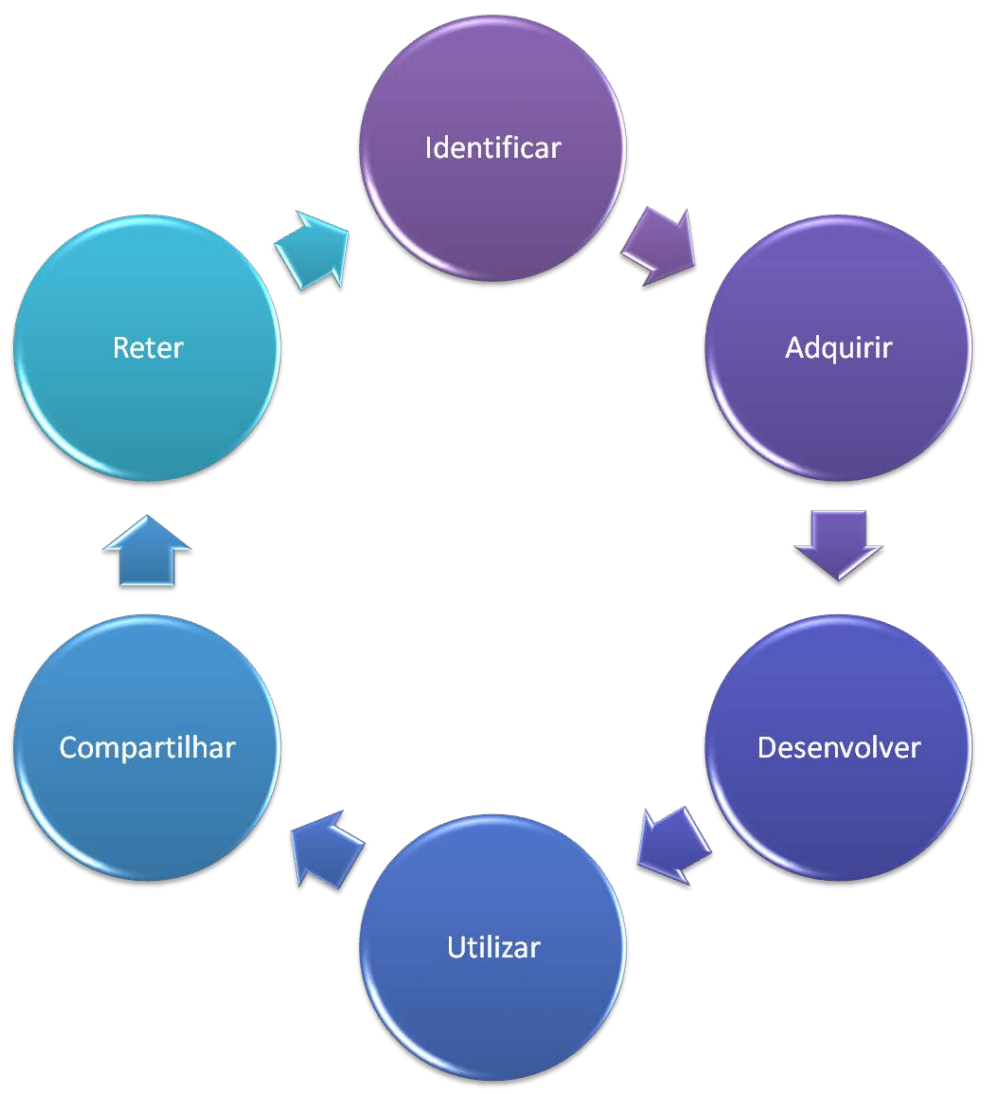

Figura 1 - Ciclo dos processos de GC identificados na UH

Fonte: Elaborada pelos autores (2016). 
Com base na análise dos dados e levando em conta o ciclo de GC proposto, considera-se importante descrever cada processo e demonstrá-lo na respectiva figura, com base no cenário de estudo.

\section{Processo de identificação do conhecimento para UH}

Momento no qual o gestor do conhecimento identifica na equipe quais os conhecimentos existentes nesse grupo para atender à demanda advinda dos pacientes. Assim, é realizada a identificação dos pontos fortes e fracos da equipe no que diz respeito ao conhecimento.

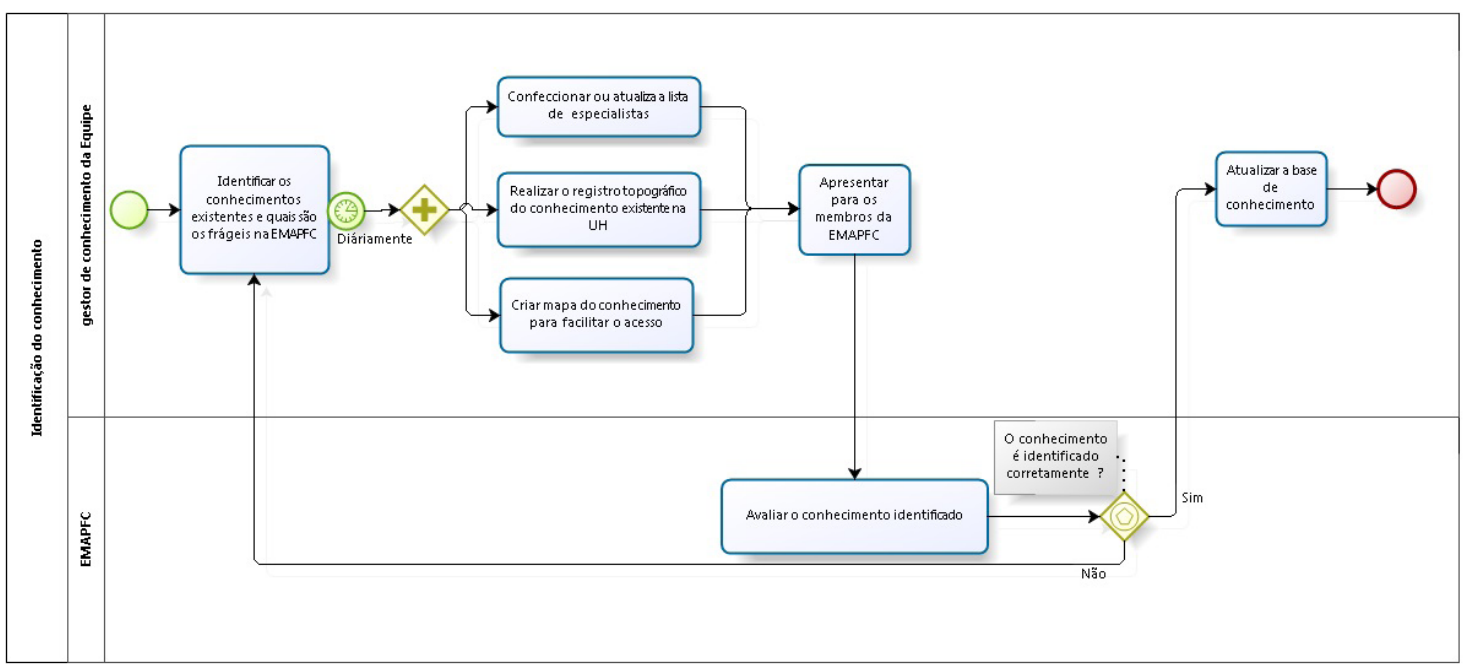

Figura 2 - Processo de identificação do conhecimento Fonte: Elaborada pelos autores (2016).

\section{Processo de aquisição do conhecimento para UH}

Processo no qual o conhecimento existente é identificado como frágil, e procede-se a busca de modos que possam suprir essa lacuna. Na prática, incluem-se a contratação de profissional que ministre palestras ou curso que venham a suprir essa fragilidade, a participação em eventos sobre o tema, e ainda a compra de materiais didáticos que atendam à demanda existente.

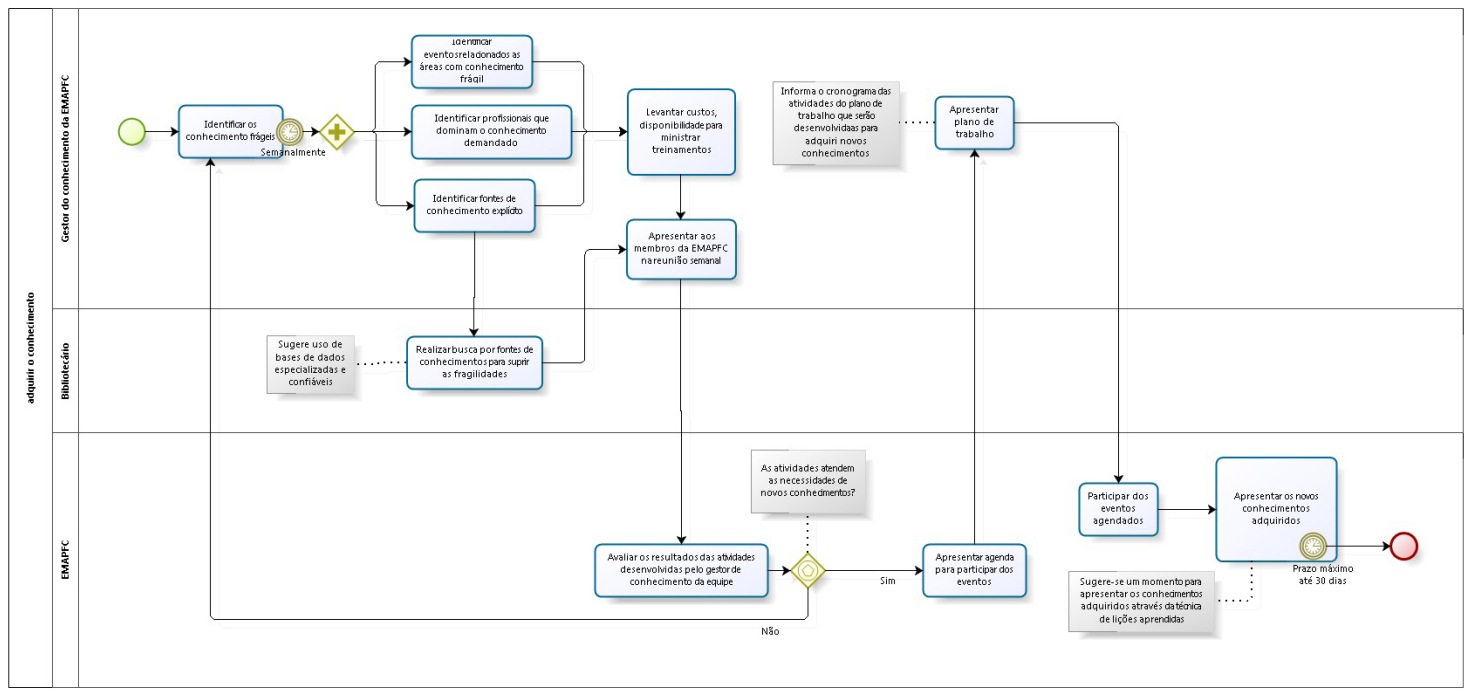

Figura 3 - Processo de aquisição do conhecimento

Fonte: Elaborada pelos autores (2016). 


\section{Processo de desenvolvimento do conhecimento para UH}

Processo em que, após a identificação da necessidade de adquirir novos conhecimentos, a equipe passa a desenvolver novas práticas que resultam em novos conhecimentos sobre como melhor atender ao paciente.

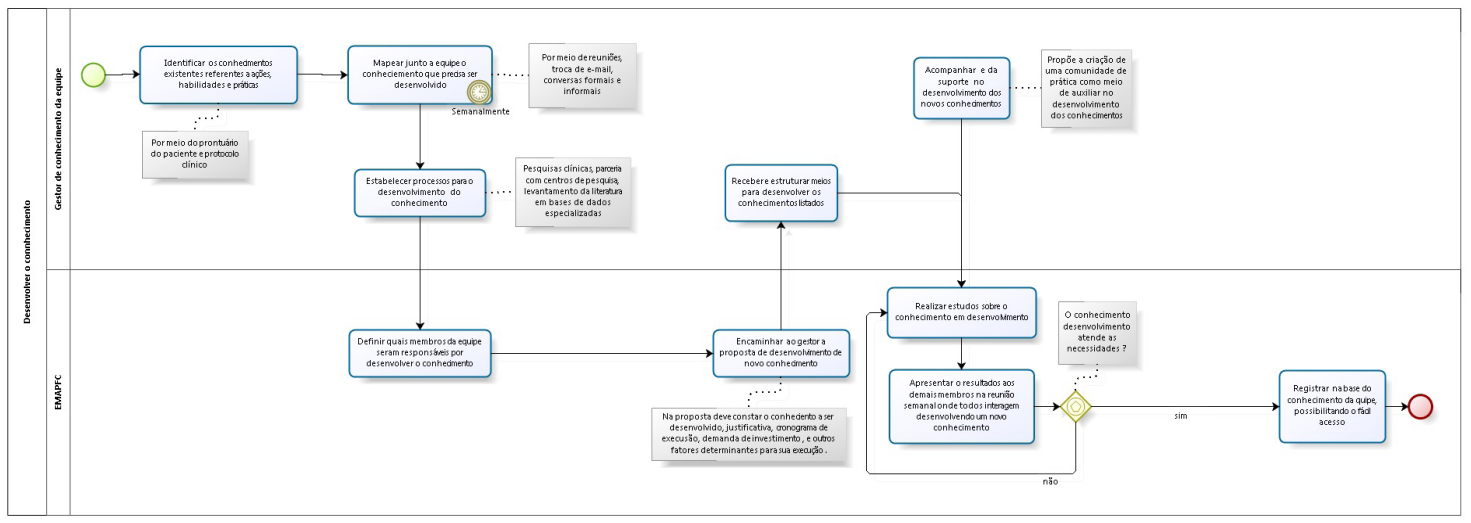

Figura 4 - Processo de desenvolvimento do conhecimento

Fonte: Elaborada pelos autores (2016).

\section{Processo de utilização do conhecimento para UH}

Processo no qual, após identificação de necessidade de novo conhecimento, os membros da equipe buscam na sua base o mais apropriado para melhor atender ao paciente.

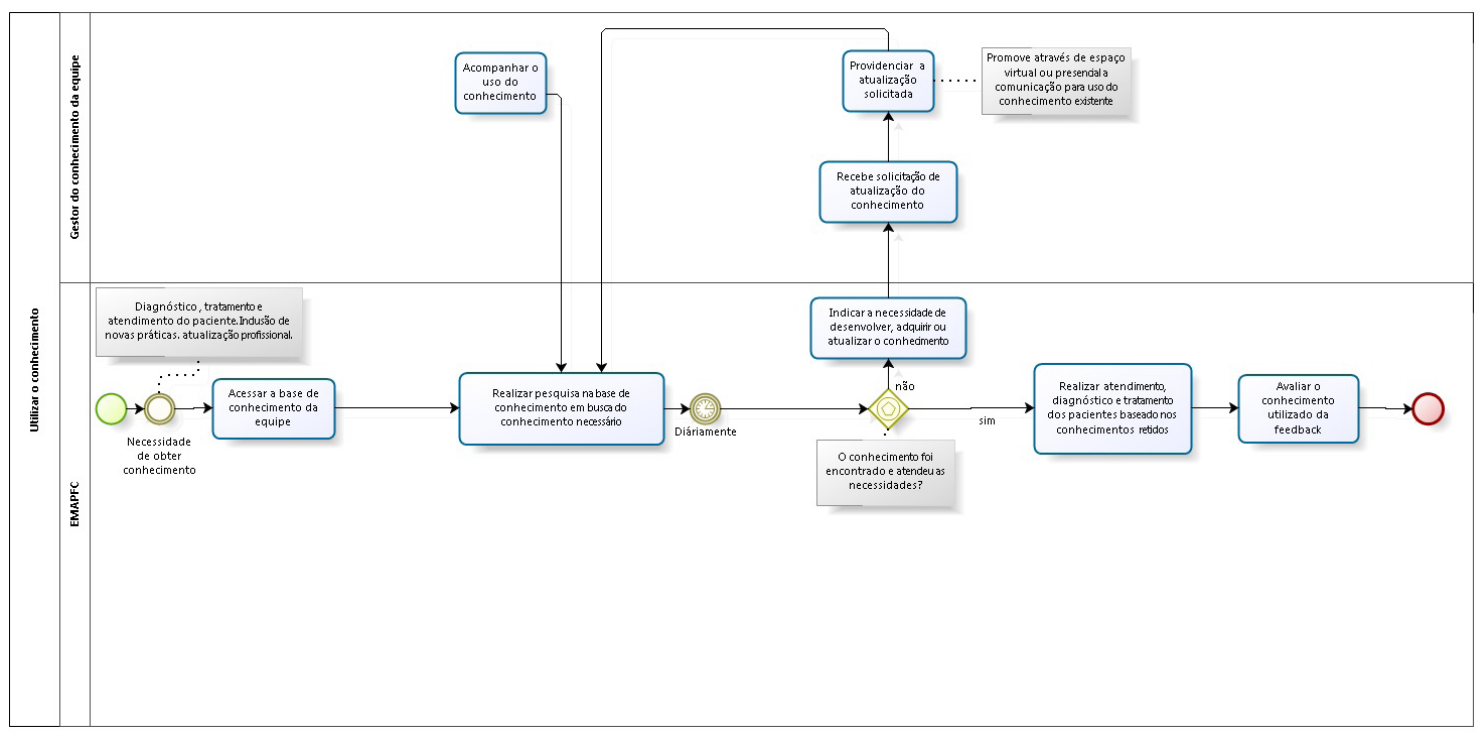

Figura 5 - Processo de utilização do conhecimento

Fonte: Elaborada pelos autores (2016).

\section{Processo de compartilhamento do conhecimento para UH}

Etapa que pode ser realizada através da participação em reuniões da equipe, compartilhando conhecimento adquirido em eventos ou através de lista de discussão por ela elaborada. Esta etapa é acompanhada pelo gestor do conhecimento que, entre suas atribuições, necessita garantir a qualidade do conhecimento a ser compartilhado bem como verificar a necessidade de melhorias do processo. 


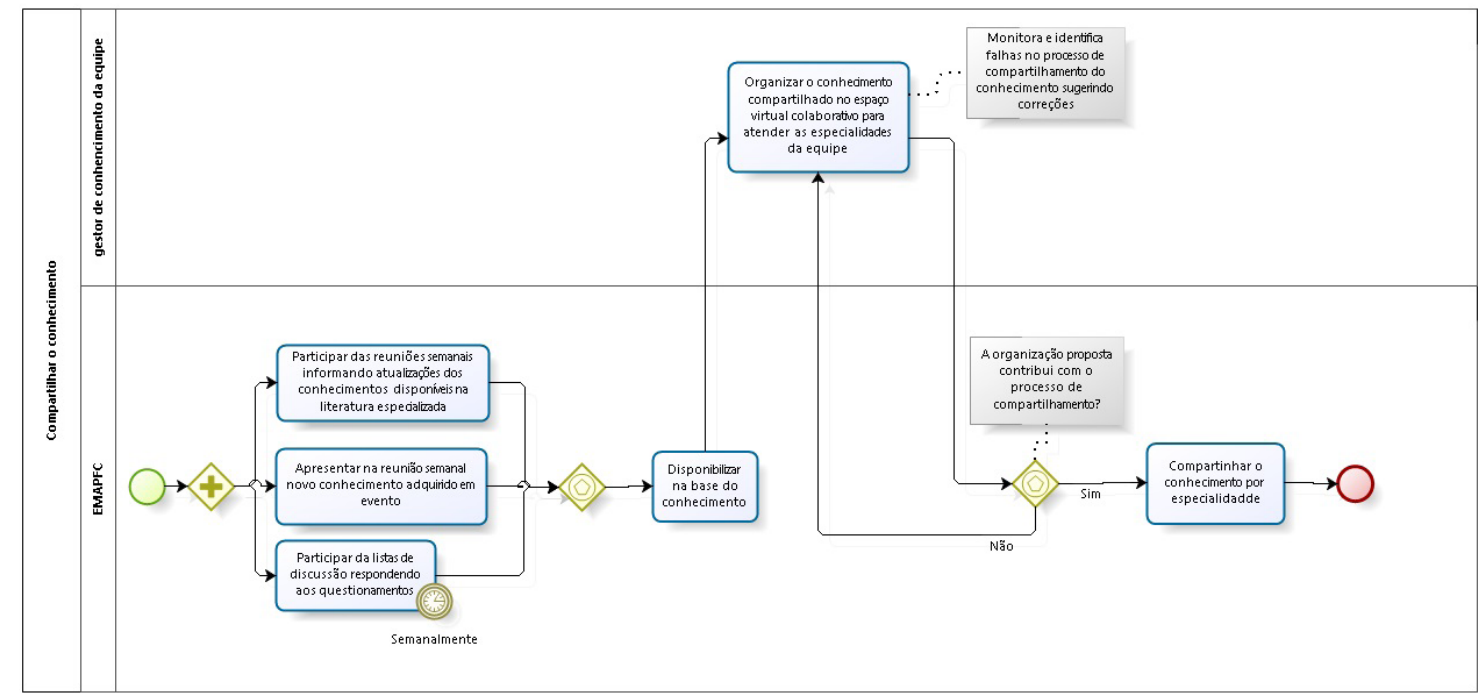

Figura 6 - Processo de compartilhamento do conhecimento Fonte: Elaborada pelos autores (2016).

\section{Processo de retenção do conhecimento para UH}

Etapa em que, após o estabelecer os critérios e as categorias para retenção do conhecimento, os membros da equipe selecionam os mais adequados e os disponibilizam na base de conhecimento.

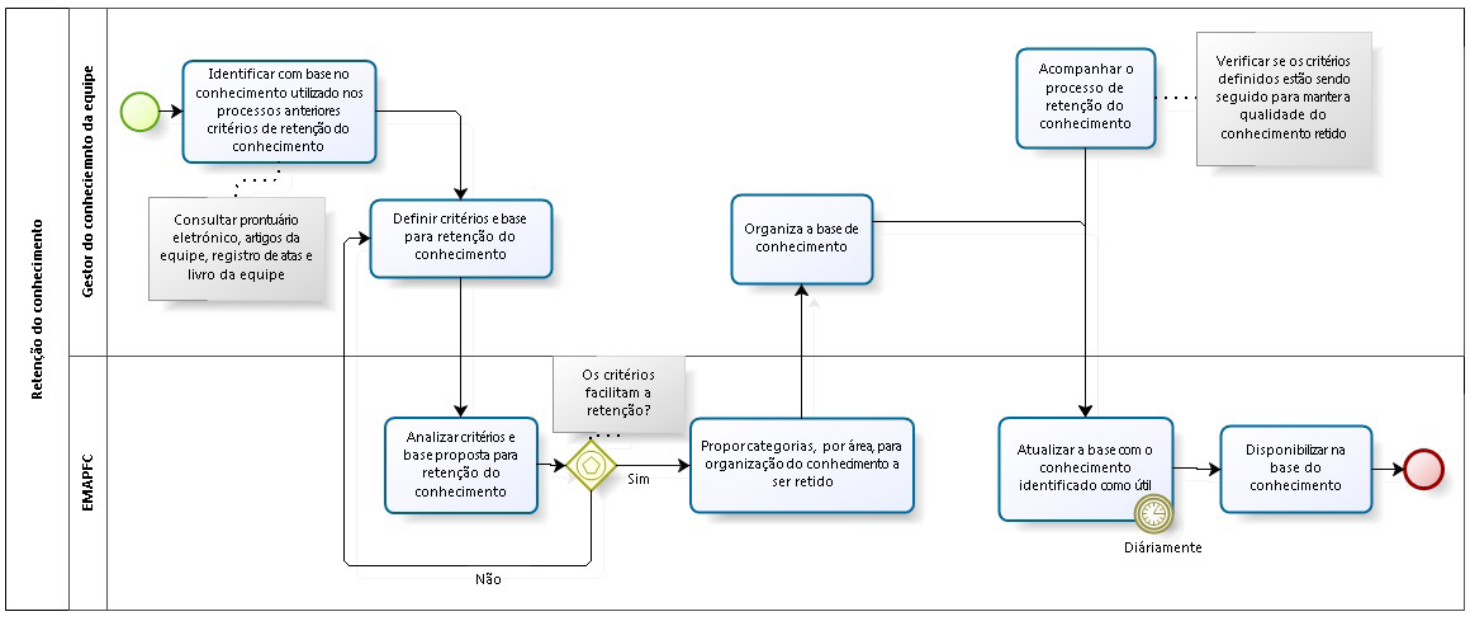

Figura 7 - Processo de retenção do conhecimento Fonte: Elaborada pelos autores (2016).

Assim, o modelo sugerido apresenta-se como prática que permite potencializar o atendimento ao paciente de fibrose cística, contribuindo com a melhoria da qualidade do serviço prestado por toda a equipe multiprofissional. 


\section{Considerações finais}

Diante da importância da prática de GC para a saúde e a discussão incipiente no Brasil, evidenciou-se a necessidade de um modelo de gestão do conhecimento para apoiar a prática clínica em uma unidade hospitalar. Para tanto se buscou compreender os modelos de GC existentes, levantando as definições e práticas existentes, o que possibilitou identificar os processos de GC relacionados às práticas clínicas na UH. Através de entrevistas, os respondentes apontaram a existência das práticas de GC, o que tornou possível estabelecer um grau de prioridade das práticas analisadas a fim de se definir um modelo de GC em que se consideram como essenciais seis processos - identificação, aquisição, desenvolvimento, utilização, compartilhamento e retenção do conhecimento - que acontecem de modo cíclico e continuado.

Por fim, destaca-se que o modelo proposto reflete as circunstâncias particulares da UH em análise, e que não se pretende generalizá-lo para outros universos, mas sim apresentar um modelo de GC crível e potencialmente transferível a outra UH. Os resultados deste trabalho reforçam o pressuposto central da pesquisa: o de que a GC em UH possibilita a melhoria das práticas clínicas, constituindo, desta maneira, uma inovação e consequentemente um desenvolvimento profissional bem como um ganho indireto para os pacientes atendidos.

\section{Referências}

1. Davila GA, Fraga BD, Diana JB, Spanhol FJ. O ciclo de gestão do conhecimento na prática: um estudo nos núcleos empresariais catarinenses. Int J Knowl Eng Manage [Internet]. 2015 fev. [citado em 2016 out. 25];2(3):43-64. Disponível em: http://goo.gl/s5CjXy .

2. Ponchirolli O, Fialho FAP. Gestão estratégica do conhecimento como parte da estratégia empresarial. R FAE [Internet]. 2005 jan./jun. [citado em 2016 out. 25];8(1):127-38. Disponível em: http://goo.gl/saiHb1. p. 130.

3. Cegarra-Navarro J-G, Cepeda-Carrión G. How to implement a knowledge management program in hospital-in-the-home units. Leadership Hlth Serv. 2010;4(23):46-56.

4. Liu FC, Cheng K-L, Chao M, Tseng H-M. Team innovation climate and knowledge sharing among healthcare managers: mediating effects of altruistic intentions. Chang Gung Med J. 2012; 35(5):408-12.

5. Nonaka I, Takeuchi H. Nonaka I, Takeuchi H . Gestão do conhecimento. Tradução de Ana Thorell. Porto Alegre: Bookman; 2008. cap. 3 Teoria da criação do conhecimento organizacional.

6. Myllärniemi J, Laihonen $\mathrm{H}$, Karppinen $\mathrm{H}$, Seppänen $\mathrm{K}$. Knowledge management practices in healthcare services. Measuring Bus Excell [Internet] 2012 [citado em 2015 nov. 25];16(4):55-65. Disponível em: http://www.emeraldinsight.com/doi/abs/10.1108/13683041211276447

7. Rowley J. The wisdom hierarchy: representations of the DIKW hierarchy. J Inf Sci. 2007;33(2)163-80.

8. Borba GS, Kliemann Neto FJ. Gestão hospitalar: identificação das práticas de aprendizagem existentes em hospitais. Saúde e Sociedade. [Internet] 2008 jan./mar. [citado em 2015 nov. 25];17(1):44-60. Disponível em: http://goo.gl/94pFMs

9. Batista FF. Modelo de gestão do conhecimento para a administração pública brasileira: como implementar a gestão do conhecimento para produzir resultados em benefício do cidadão. Brasília: Ipea; 2012.

10. Armoogum K, Buchgeister M. Factors affecting knowledge sharing in the radiotherapy department: the radiation physics team as a community of practice. ] Radiother Pract [Internet]. 2010 Mar [citado em 2015 out 27];9(1):17-26. Disponível em: http://goo.gl/abtFF3.

11. Macedo M et al. Gestão do conhecimento organizacional. Florianópolis: UFSC; 2010. p. 40.

12. Mattera TC Gestão do conhecimento na prática. In: Souto LF, organizador. Gestão da informação e do conhecimento: práticas e reflexões. Rio de Janeiro: Interciência; 2014.

13. Davenport TH; Prusak L. Conhecimento empresarial: como as organizações gerenciam o seu capital intelectual. Rio de Janeiro: Campus; 1998. 
14. Gnecco Júnior L, Pereira MF, Dalmau MBL, Santana JQ, Tecchio EL. Gestão do conhecimento: fatores críticos de sucesso. Reuna [Internet]. 2010 [citado em 2015 nov, 22];15(1):47-64. Disponível em: http://goo.gl/Gfhofn.

15. Herrera CGN. El liderazgo en la gestión del conocimiento [Internet]. 2010 [citado em 2016 fev. 10]. Disponível em: http://goo.gl/fj7gEk

16. Nonaka I, Takeuchi H. Criação de conhecimento na empresa: como as empresas japonesas geram a dinâmica da inovação. Rio de Janeiro: Campus; 1997.

17. Von Krog G; Ichijo K; Nonaka I. Facilitando a criação de conhecimento: reinventando a empresa com o poder da inovação contínua. Rio de Janeiro: Campus; 2001.

18. Choo CW. A organização do conhecimento: como as organizações usam a informação para criar significado, construir conhecimento e tomar decisões. São Paulo: Senac; 2003.

19. Teixeira Filho J. Gerenciando conhecimento: como a empresa pode usar a memória organizacional e a inteligência competitiva no desenvolvimento dos negócios. Rio de Janeiro: Senac; 2000.

20. Probst G, Raub S, Romhardt K. Gestão do conhecimento: os elementos construtivos do sucesso. Porto Alegre: Bookman; 2002.

21. Nonaka I, Peltokorpi V. Objectivity and subjectivy in knowledge: a review of 20 top articles. Knowl Process Manag [Internet]. 2006 Apr/June [citado 2016 fev. 06]. Disponível em: http://onlinelibrary.wiley. com/doi/10.1002/kpm.251/abstract.

22. Alvarenga Neto RCD. Gestão do conhecimento em organizações: proposta de mapeamento conceitual integrativo. São Paulo: Saraiva; 2008.

23. Dalkir, K. Knowledge management in theory and practice. Boston: Elsevier; 2005.

24. Cavalcante J. Análise das práticas de gestão do conhecimento em uma empresa de serviços de assessoria e educação profissional. Qualit@s [Internet]. 2011 [citado em 2016 fev. 20]:12(2):1-17. Disponível em: http://goo.gl/OBIJ39.

25. Pacheco JLC. Análise do setor de planos de saúde: um estudo de caso com operadoras do Rio de Janeiro [dissertação]. Niterói: Universidade Federal Fluminense; 2005.

26. Bem RM, Prado ML, Delfino N. Desafios à implantação da gestão do conhecimento: a questão cultural nas organizações públicas federais brasileiras. RDBCI [Internet]. 2013 [citado em 2016 fev. 22]:11(2):123-135. Disponível em: http://goo.gl/wW9F52.

27. Valentim MLP. Gestão da informação e do conhecimento em ambientes organizacionais. TPBCI [Internet]. 2008 [citado em 2015 nov. 02]:1(1). Disponível em: http://inseer.ibict.br/ancib/index.php/ tpbci/article/view/110/151

28. Young R, editor. Knowledge management: tools and techniques manual [Internet]. Toquio: Asian Productivity Organization; 2010 [citado em 2016 fev. 25]. Disponível em: http://goo.gl/L2B8Md.

29. Sumet S, Suwannapong N, Howteerakul N, Thammarat C. Knowledge management model for quality improvement in the homodialysis unit of a non-profit private hospital, Bangkok, Thailand. Leadership HIth Serv [Internet] 2012 [citado em 2015 dez. 02];25(4):306-17. Disponível em: http://goo.gl/umSBbG

30. Elwyn G, Taubert M, Kowalczuk J. Sticky knowledge: a possible model for investigating implementation in healthcare contexts. Implementation Sci [Internet] 2007 [citado em 2016 jan. 20];44(2). Disponível em: http://goo.gl/IgEdxh.

31. Laihonen H. Knowledge structures of a health ecosystem. J Health Organ Manag [Internet] 2012 [citado em 2015 nov. 05];26(4). Disponível em: http://goo.gl/IoYbuo

32. Creswell JW. Projeto de pesquisa: métodos qualitativo, quantitativo e misto. 2 ed. Porto Alegre: Artmed; 2010. p.73 e 76.

33. Lefevre F, Lefevre AMC. Pesquisa de representação social: a metodologia do discurso do sujeito coletivo. Brasilia: Liber Livor; 2012. p. 31.

34. Santaela, L. Comunicação e pesquisa: projetos para mestrado e doutorado. 2 ed. São José do Rio Preto, SP: Bluecom Comunicação; 2010. 